\title{
A note on the recursive calculation of dominant sin- gular subspaces
}

\author{
Nicola Mastronardi, Marc Van Barel, and Raf Vandebril
}

Nicola Mastronardi Istituto per le Applicazioni del Calcolo M. Picone, sez. Bari, Italy N.Mastronardi@ba.iac.cnr.it

Marc Van Barel
Department of Computer Science
KU Leuven, Belgium
Marc.VanBarel@cs.kuleuven.be
Raf Vandebril
Department of Computer Science
KU Leuven, Belgium
Raf.Vandebril@cs.kuleuven.be

\begin{abstract}
A recursive procedure for computing an approximation of the left and right dominant sin- gular subspaces of a given matrix is proposed in [1]. The method is particularly suited for matrices with many more rows than columns. The procedure consists of a few steps. In one of these steps a Householder transformation is multiplied to an upper triangular matrix. The following step consists in recomputing an upper triangular matrix from the latter product. In [1] it is said that the latter step is accomplished in $O\left(k^{3}\right)$ operations, where $\mathrm{k}$ is the order of the triangular matrix. In this short note we show that this step can be accomplished in $O\left(k^{2}\right)$ operations.
\end{abstract}

\section{Article information}

- Mastronardi, Nicola; Van Barel, Marc; Vandebril, Raf. A note on the recursive calculation of dominant singular subspaces, Numerical Algorithms, volume 38, issue 4, pages 237-242, 2005.

- The content of this article is identical to the content of the published paper, but without the final typesetting by the publisher.

- Journal's homepage: http://link.springer.com/journal/11075

- Published version: http://dx.doi.org/10.1007/s11075-004-4338-x

- KU Leuven's repository url: https://lirias.kuleuven.be/handle/123456789/124943

\section{KU LEUVEN}




\title{
A note on the recursive calculation of dominant singular subspaces*
}

\author{
Nicola Mastronardi ${ }^{\mathrm{a}}$, Marc Van Barel $^{\mathrm{b}}$ and Raf Vandebril ${ }^{\mathrm{b}}$ \\ a Istituto per le Applicazioni del Calcolo “M. Picone”, Consiglio Nazionale delle Ricerche, \\ via G. Amendola 122/D, I-70126 Bari, Italy \\ E-mail: n.mastronardi@area.ba.cnr.it \\ ${ }^{b}$ Department of Computer Science, Katholieke Universiteit Leuven, Celestijnenlaan 200A, \\ B-3001 Leuven (Heverlee), Belgium \\ E-mail: \{Marc.VanBarel;Raf.Vandebril\}@cs.kuleuven.ac.be
}

Received 7 June 2004; accepted 10 September 2004

Communicated by P. Van Dooren

\begin{abstract}
A recursive procedure for computing an approximation of the left and right dominant singular subspaces of a given matrix is proposed in [1]. The method is particularly suited for matrices with many more rows than columns. The procedure consists of a few steps. In one of these steps a Householder transformation is multiplied to an upper triangular matrix. The following step consists in recomputing an upper triangular matrix from the latter product. In [1] it is said that the latter step is accomplished in $\mathrm{O}\left(k^{3}\right)$ operations, where $k$ is the order of the triangular matrix. In this short note we show that this step can be accomplished in $\mathrm{O}\left(k^{2}\right)$ operations.
\end{abstract}

Keywords: Householder matrix, Givens matrix, QR factorization, URV factorization, updating, singular value decomposition

AMS subject classification: 15A15, 15A09, 15A23

\footnotetext{
* This research was partially supported by MIUR, grant number 2002014121 (first author) and by the Research Council K.U.Leuven, project OT/00/16 (SLAP: Structured Linear Algebra Package), by the Fund for Scientific Research-Flanders (Belgium), projects G.0078.01 (SMA: Structured Matrices and their Applications), G.0176.02 (ANCILA: Asymptotic aNalysis of the Convergence behavior of Iterative methods in numerical Linear Algebra), G.0184.02 (CORFU: Constructive study of Orthogonal Functions) and G.0455.0 (RHPH: Riemann-Hilbert problems, random matrices and Padé-Hermite approximation), and by the Belgian Programme on Interuniversity Poles of Attraction, initiated by the Belgian State, Prime Minister's Office for Science, Technology and Culture, project IUAP V-22 (Dynamical Systems and Control: Computation, Identification \& Modelling) (second and third author). The scientific responsibility rests with the authors.
} 


\section{Introduction}

In [1], a recursive procedure is designed for computing an approximation of the left and right dominant singular subspaces of a matrix, whose columns are produced incrementally. The method is particularly suited for matrices with many more rows than columns. The procedure consists of a few steps. In one of these steps a Householder transformation is multiplied to an upper triangular matrix. The following step consists in recomputing an upper triangular matrix from the latter product. In [1] it is said that the latter step is accomplished in $\mathrm{O}\left(k^{3}\right)$ operations, where $k$ is the order of the triangular matrix. In this short note we show that this step can be accomplished in $\mathrm{O}\left(k^{2}\right)$ operations. Moreover, the exploitation of the matrix structure in the latter step allows to reduce the computational complexity from $\mathrm{O}\left(k^{3}\right)$ to $\mathrm{O}\left(k^{2}\right)$ if also the right dominant singular subspace is required.

The note is organized as follows. In section 2 the algorithm proposed in [1] is described and the proposed modification is described in section 3.

\section{Recursive calculation of dominant singular subspaces}

In this section we shortly describe the algorithm proposed in [1] to recursively compute dominant singular subspaces, whose columns are known incrementally, i.e., at each step one more column is added to the matrix. The algorithm is particularly suited for matrices $A \in \mathbb{R}^{m \times n}, m \gg n$.

At each recursion, the following steps are performed:

Step 1. Known the orthogonal matrix $U \in \mathbb{R}^{m \times k}$, and the upper triangular matrix $R \in \mathbb{R}^{k \times k}$ of the $U R V$ factorization of the matrix $\tilde{A} \in \mathbb{R}^{m \times k}, k<n \ll m$, $\tilde{A}=U R V$, with $V \in \mathbb{R}^{k \times k}$ orthogonal ( $V$ does not need to be known), a new column $b$ is added to $\tilde{A}$,

$$
\hat{A}=[\tilde{A}, b] .
$$

Step 2. Update the $U R V$ factorization of the extended matrix $\hat{A}$ via Gram-Schmidt orthogonalization,

$$
\begin{aligned}
r & =U^{\mathrm{T}} b, \\
\hat{b} & =b-U r, \\
\rho & =\|\hat{b}\|_{2}, \\
\hat{u} & =\frac{b}{\rho} .
\end{aligned}
$$

Then

$$
[\tilde{A} \mid b]=[U R \mid b]\left[\begin{array}{l|l}
V \mid & 1
\end{array}\right]=[U \mid \hat{u}]\left[\begin{array}{l|l}
R & r \\
\hline
\end{array}\right]\left[\begin{array}{l|l}
V & \mid \\
\hline & 1
\end{array}\right]=[U \mid \hat{u}] \widehat{R}\left[\begin{array}{l|l}
V & \\
\hline & 1
\end{array}\right] .
$$


Step 3. Let $\sigma_{k+1}$ be the smallest singular value of $\widehat{R}$ and $u_{k+1}$ the corresponding left singular vector. Let $G_{u}$ be an orthogonal matrix such that

$$
G_{u}^{\mathrm{T}} u_{k+1}=e_{k+1}=[0, \ldots, 0,1]^{\mathrm{T}},
$$

and compute

$$
G_{u}^{\mathrm{T}} \widehat{R}=\widehat{S},
$$

with $\widehat{S} \in \mathbb{R}^{(k+1) \times(k+1)}$ no more upper triangular.

Step 4. Compute the $R Q$ factorization of $\widehat{S}$,

$$
\widehat{S}=\widetilde{R} \widetilde{H},
$$

where $\widetilde{R}$ and $\widetilde{H}$ are upper triangular and orthogonal matrices of order $(k+1)$, respectively. It turns out that

$$
\widetilde{R}=\left[\begin{array}{l|l}
R_{n} & \\
\hline & \sigma_{k+1}
\end{array}\right] .
$$

Step 5. Update the orthogonal matrix $U$

$$
U \leftarrow U G_{u}, \quad V \leftarrow \widetilde{H}^{\mathrm{T}} V,
$$

and set

$$
\tilde{A}=U(:, 1: k) R_{n} V(1: k,:)
$$

Remark. It is not necessary to update he matrix $V$, as stated in [1], if only the left dominant singular subspaces are needed.

The matrix $G_{u}$ in step 3 could be either a product of $k$ Givens rotations or a Householder matrix. If the product of $k$ Givens rotations is chosen, step 4 can be accomplished in $\mathrm{O}\left(k^{2}\right)$ operations in a way similar to the one described in [2], and the matrix $\widetilde{H}$ is given by the product of $k$ different Givens rotations.

However, the costly part of the algorithm is the update of the matrix $U$, and hence it is preferable to choose $G_{u}$ to be a Householder transformation. In fact, the cost of the latter product $U G_{u}$ is $4 m k+2 m$ if $G_{u}$ is a Householder transformation, whereas the cost is $6 m k$ if the orthogonal matrix $G_{u}$ is given by the product of $k$ Givens rotations. In [1] is said that the price to pay choosing $G_{u}$ a Householder transformation is that the $R Q$ factorization in step 4 is computed in $\mathrm{O}\left(k^{3}\right)$ operations.

In the next section we show that the latter factorization can be accomplished in $\mathrm{O}\left(k^{2}\right)$ operations. 


\section{Computation of the $R Q$ factorization of the matrix $\widehat{S}$}

Let $G_{u}=I-\beta z z^{\mathrm{T}}$ be the Householder transformation such that $G_{u}^{\mathrm{T}} u_{k+1}=e_{k+1}$. Let us denote by $\hat{s}_{i}, i=1, \ldots, k+1$, the $i$ th column of $\widehat{S}$. Similarly, we use the notation $\hat{r}_{i}$ for the $i$ th column of the matrix $\widehat{R}$.

The product $G_{u}^{\mathrm{T}} \widehat{R}$ can be written as

$$
G_{u}^{\mathrm{T}} \widehat{R}=\left[G_{u}^{\mathrm{T}} \hat{r}_{1}, G_{u}^{\mathrm{T}} \hat{r}_{2}, \ldots, G_{u}^{\mathrm{T}} \hat{r}_{k+1}\right] .
$$

We observe that

$$
G_{u}^{\mathrm{T}} \hat{r}_{i}=\left(I-\beta z z^{\mathrm{T}}\right) \hat{r}_{i}=\hat{r}_{i}-\left(\beta z^{\mathrm{T}} \hat{r}_{i}\right) z .
$$

Since $\widehat{R}$ is an upper triangular matrix, the $i$ th column of $\widehat{S}$ below the main diagonal is a multiple of $z$, i.e.,

$$
\hat{s}_{i}(i+1: k+1)=\left(G_{u}^{\mathrm{T}} \hat{r}_{i}\right)(i+1: k+1)=-\left(\beta z^{\mathrm{T}} \hat{r}_{i}\right) z(i+1: k+1) .
$$

In other words, the strictly lower triangular part of $\widehat{S}$ is the strictly lower triangular part of a rank-one matrix. Therefore, the retriangularization of $\widehat{S}$ can be accomplished applying only $2 k-1$ Givens rotations to the right, with $\mathrm{O}\left(k^{2}\right)$ operations as described below.

We observe that the strictly lower triangular part is not explicitly computed, since the entries of the $i$ th column below the main diagonal are given by $-\beta z^{\mathrm{T}} \hat{r}_{i} z(k), k>i$, where $z(k)$ is the $k$ th entry of the vector $z$. Therefore, for any column it is only needed to store the values $\gamma(i)=\beta z^{\mathrm{T}} \hat{r}_{i}, i=1, \ldots, k$.

Let us suppose, for simplicity, that $k=5$, and denote with $\square$ the entries of $\widehat{S}$ belonging to the strictly lower triangular part:

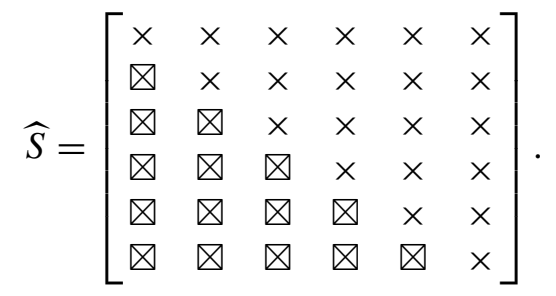

Applying a Givens rotation $G_{1}$ to the right in order to annihilate the entry $(3,1)$, the whole column below this entry is annihilated, since both the columns are a multiple of the vector $z$ below the main diagonal:

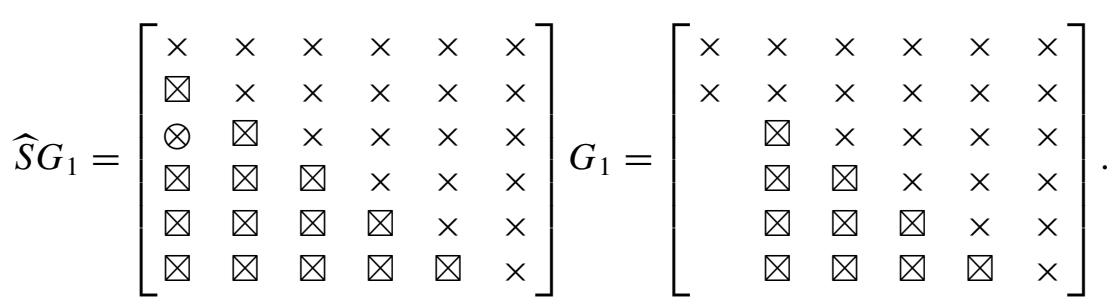


Let $c_{1}$ and $s_{1}$ be the Givens coefficients of $G_{1}$, i.e.,

$$
G_{1}=\left[\begin{array}{cc}
c_{1} & s_{1} \\
-s_{1} & c_{1}
\end{array}\right], \quad c_{1}=\frac{z^{\mathrm{T}} \hat{r}_{2}}{\sqrt{\left(z^{\mathrm{T}} \hat{r}_{1}\right)^{2}+\left(z^{\mathrm{T}} \hat{r}_{2}\right)^{2}}}, \quad s_{1}=\frac{z^{\mathrm{T}} \hat{r}_{1}}{\sqrt{\left(z^{\mathrm{T}} \hat{r}_{1}\right)^{2}+\left(z^{\mathrm{T}} \hat{r}_{2}\right)^{2}}} .
$$

Of course, it is not necessary to compute $\widehat{S} G_{1}$ below the upper Hessenberg part of the matrix. In fact the entries of the first column with row indexes greater than 2 are zero. Moreover, the entries of the second column with row indexes greater than 2 are given by

$$
\beta z(i) \sqrt{\left(z^{\mathrm{T}} \hat{r}_{1}\right)^{2}+\left(z^{\mathrm{T}} \hat{r}_{2}\right)^{2}}, \quad i>2 .
$$

Therefore it is only needed to update the value $\gamma(2)=\beta \sqrt{\left(z^{\mathrm{T}} \hat{r}_{1}\right)^{2}+\left(z^{\mathrm{T}} \hat{r}_{2}\right)^{2}}$.

In the same manner, applying a Givens rotation $G_{2}$ to the right in order to annihilate the entry $(4,2)$, the whole row below this entry is annihilated.

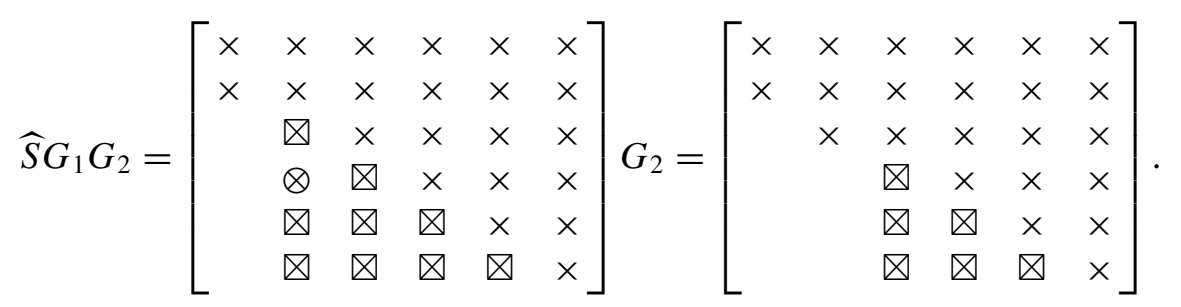

Also in this case, only the upper Hessenberg part of columns 2 and 3 and the coeffcient $\gamma(3)$ need to be updated.

Proceeding in this way, after $k-1$ multiplications to the right by Givens rotations, the original matrix is transformed into an upper Hessenberg one with $\mathrm{O}\left(k^{2}\right)$ operations,

$$
\widehat{S} G_{1} G_{2} \cdots G_{k-1}=\left[\begin{array}{cccccc}
\times & \times & \times & \times & \times & \times \\
\times & \times & \times & \times & \times & \times \\
& \times & \times & \times & \times & \times \\
& \times & \times & \times & \times \\
& & \times & \times & \times \\
& & & \times & \times
\end{array}\right]
$$

To reduce the latter Hessenberg matrix to upper triangular form, apply $k$ Givens rotations $\widehat{G}_{i}, i=k, k-1, \ldots, 2,1$, to the right in order to annihilate the entries $(k+1, k)$, $(k, k-1), \ldots,(2,1)$. The whole transformation is depicted in figure 1 . Therefore the reduction of the matrix $\widehat{S}$ into triangular form can be made in $\mathrm{O}\left(k^{2}\right)$ operations.

If one is interested also in the right singular dominant subspace, the latter construction of the $R Q$ factorization of the matrix $\widehat{S}$ allows to reduce the computational complexity from $\mathrm{O}\left(k^{3}\right)$ to $6 k^{2}$ flops because the updated matrix of the right singular vectors is given by

$$
Q\left[\begin{array}{l|l}
V & \\
\hline & 1
\end{array}\right]=\widehat{G}_{1}^{\mathrm{T}} \widehat{G}_{2}^{\mathrm{T}} \cdots \widehat{G}_{k-1}^{\mathrm{T}} \widehat{G}_{k}^{\mathrm{T}} G_{k-1}^{\mathrm{T}} G_{k-2}^{\mathrm{T}} \cdots G_{2}^{\mathrm{T}} G_{1}^{\mathrm{T}}\left[\begin{array}{l|l}
V & \\
\mid & 1
\end{array}\right] .
$$




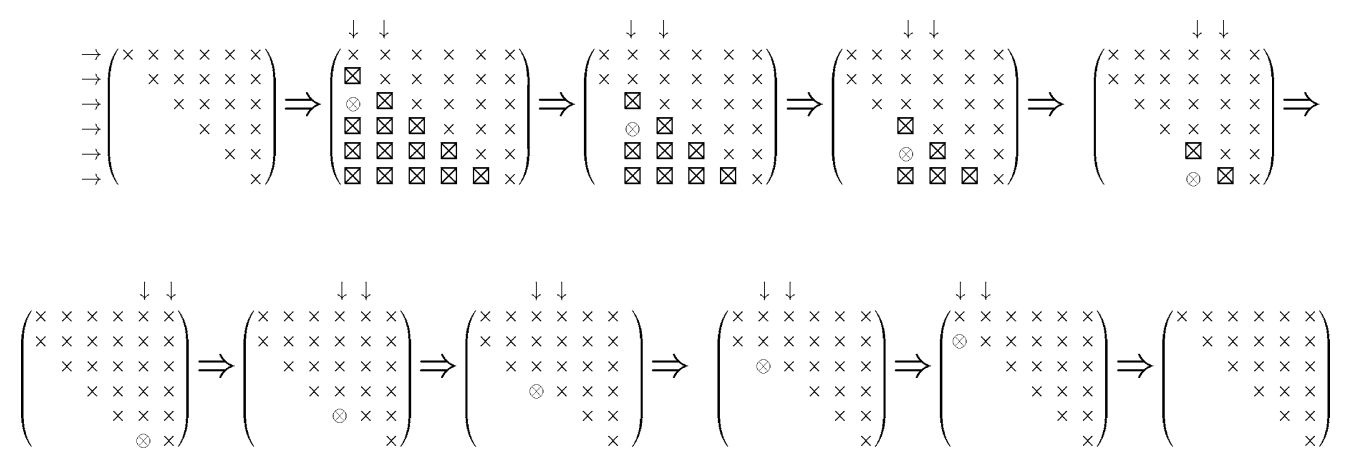

Figure 1 . Multiplication of $\widehat{R}$ to the left by a Householder transformation followed by a sequence of $2 k-1$ multiplications by Givens rotations to the right in order to retrieve the upper triangular structure. With $\otimes$ is denoted the entry of the matrix to be annihilated by a Givens transformation.

\section{References}

[1] Y. Chahlaoui, K. Gallivan and P. Van Dooren, Recursive calculation of dominant singular subspaces, SIAM J. Matrix Anal. Appl. 25(2) (2004) 445-463.

[2] T.F. Chan, An improved algorithm for computing the singular value decomposition, ACM Trans. Math. Software 8(1) (1982) 72-83. 